\title{
Come ottimizzare il trattamento dopo la fase acuta: quali programmi per quali pazienti?
}

\author{
G. Sinagra
}

Monaldi Arch Chest Dis 2006; 66: 133-135.

U.O. Cardiologia, Trieste.

Desidero che la mia presentazione sia solo di spunto per costruire percorsi per il paziente postacuto, in termini di quali programmi e per quali pazienti. A mio avviso, la lettura dei documenti delle linee guida recentemente prodotte, combinati con il documento ANMCO "Struttura e Organizzazione Funzionale in Cardiologia" e il documento di Consenso che l'ANMCO-Area Scompenso-Management si accinge a definire nel suo testo alla fine ci aiuteranno a comprendere, anche rispetto al paziente che ha avuto un passaggio di terapia intensiva o di instabilizzazione acuta, quali siano le tappe da percorrere. Come clinici, dovremo essere sempre più attenti allo specifico profilo clinico dei nostri pazienti per una corretta applicazione delle Linee Guida che induca "buona medicina" [1]. In questo senso, i registri dell'ANMCO e della Rete sullo Scompenso Cronico e quelli generati del Registro sullo Scompenso Acuto ci aiutano a capire "quali profili" per "quali comportamenti”. La mortalità per scompenso cardiaco cronico si è ridotta; questo dato probabilmente riguarda anche lo scompenso cardiaco acuto. Molti dei risultati osservati in termini di end point hard è il frutto dell'addizione progressiva di principi attivi alle efficaci terapie preesistenti. Ma questa addizione progressiva nella logica del trial ha dietro il rigore del disegno di uno studio e l'attenzione degli sperimentatori rispetto alle ipotesi che si testano. Nel mondo reale, una triplice terapia di addizione, specie se, nello specifico paziente, si dovesse decidere, o si dovesse ipotizzare, l'impiego per esempio, dell'antialdosteronico in associazione al sartano ed ACE-inibitore (paziente in avanzata classe NYHA), non è detto che generi la stessa evidenza sugli end point hard così come ottenuta nel grande trial. Un punto importante è che, anche quando per anni abbiamo stressato i passi che avevamo fatto nel miglioramento della mortalità dei pazienti con scompenso cardiaco in termini di deficit di pompa, eravamo piuttosto rassegnati nel dire come, nonostante il miglioramento dell'approccio farmacologico e la terapia dello scompenso, fossimo sostanzialmente perdenti, non solo nello stratificare i pazienti, ma anche nel valutare, e nell'influenzare, il loro rischio di morte improvvisa. Lo SCDHeFT è uno studio che, a mio avviso, è destinato a far riflettere la comunità scientifica ed a farla riflettere anche in termini di percorsi, perché non dice soltanto che il braccio terapia medica ottimizzata presenta una mortalità a 5 anni più bassa di quanto in precedenza noto per pazienti in classe NYHA II-III, ma anche che, per pazienti che abbiano una sopravvivenza sufficientemente lunga da dare il tempo alle curve di aprirsi, il defibrillatore riesce a ridurre ulteriormente il rischio di morte improvvisa. In termini di percorsi del paziente che ha superato la fase acuta, si possono identificare sostanzialmente 4 aree critiche dal punto di vista operativo. Una prima area è che la diagnosi di scompenso cardiaco, e quella acuta lo è per eccellenza, perché ci si è convinti che il paziente aveva una situazione di instabilità acuta, non può essere un punto di arrivo. Una prima area critica è, quindi, che il paziente che ha superato l'esperienza di insufficienza cardiaca acuta deve avere una definizione eziologica del suo scompenso cardiaco e, attraverso la definizione dei meccanismi che lo hanno eziologicamente determinato e che possono patogenicamente contribuire ad esso, può avere un orientamento terapeutico specifico. E inutile immaginare che tutti i pazienti possano comportarsi come un modello strutturato di cardiopatia per la quale dobbiamo contrastare i rimodellamenti, economizzare consumi di ossigeno, resincronizzare se c'è da risincronizzare, se a monte non ricordiamo che non possiamo convivere con un modello eziologicamente o patogenicamente non definito. Credo che tutti voi siate d'accordo che questa è, clinicamente, una cardiomiopatia dilatativa ma penso che siate d'accordo che, se una cardiomiopatia dilatativa ha anche una componente di insufficienza mitralica di questa entità, che al meglio del trattamento farmacologico non si modifica in maniera significativa, al giorno d'oggi diventa un modello di cardiopatia che ha avuto uno scompenso acuto e che entra in fase di gestione cronica, per la quale pensare a modelli terapeutici che potrebbero non essere necessariamente $o$ solo farmacologici. Certamente non possiamo convivere con il dubbio che quest'altro paziente scompensatosi acutamente e migliorato in terapia, apparentemente asintomatico per angina ma con ECG marcatamente patologico per ischemia, possa essere portatore di una malattia coronarica critica potenzialmente rivascolarizzabile. Quando lo scompenso cardiaco acuto è realmente acuto e iperteso, bisogna porsi il problema della malattia coronarica associata, non fosse altro che perché quel paziente è un aterosclerotico. Ma non dimentichiamo che alcuni scompensi cardiaci acuti gravemente ipertesi hanno una stenosi delle arterie renali che, finché non sarà identificata, ci farà scontrare con farmaci che peggiorano la funzione renale del paziente, non controllano lo scompenso, non evitano il rischio di nuove crisi di scompenso cardiaco. Una seconda area critica per l'inquadramento del paziente con scompenso cardiaco è la terapia farmacologica. Bisogna utilizzare meglio i farmaci di cui disponiamo sia qualita- 
tivamente che quantitativamente in termini di dosi e combinazioni dei farmaci rispetto allo specifico paziente. Le linee guida [2, 3] non potranno mai darci una risposta univoca. Per esempio, le linee guide, quando si parla di terapia con antialdosteronici, in generale tendono a non escludere i pazienti che abbiano meno di 2,5 di creatinina e meno di 5 di potassemia (ad es. quelle dell'American Heart Association 2005). Ma questo non autorizza una strategia di antialdosteronico in un paziente che è gia in triplice terapia con ace-inibitori, sartanico e betabloccante, in particolare se è anziano, e/o diabetico, e con valori di creatinina che confinano con quelli che vi ho detto. Va dunque bene utilizzare meglio le combinazioni, ma facendolo rispetto ad una personalizzazione del trattamento che, a mio avviso, non potrà essere standardizzata da logiche rigide di strutture, di percorso o di processo, rinunciando ad un approccio clinico personalizzato al paziente che continuerà a costituire, specie in una patologia che spesso coinvolge persone anziane, un momento irrinunciabile. Non aspettiamoci che la terapia produca grandi novità per alcuni anni, ad esempio per gli inibitori della renina. Alcuni importanti progressi sono stati compiuti. Questa rappresentazione per step terapeutici come approccio al Paziente scompensato è ampiamente superata in particolare in un contesto di medicina riabilitativa e di cardiologia riabilitativa. Per molti anni infatti la riabilitazione cardiologica non è stata considerata un approccio allo scompenso cardiaco. Questo destino ed ossequio a paradigmi a coinvolto anche il beta blocco. Oggi vi sono evidenze che hanno mutato consuetudini di pensiero e paradigmi comportamentali. I betabloccanti appartenevano alla fascia dei pazienti poco compromessi, e oggi sappiamo che possono appartenere, nei pazienti adeguatamente selezionati, anche a quelli gravemente compromessi; gli ARB avevano uno spazio che era sostanzialmente alternativo agli ACE-inibitori quando gli ACE non erano tollerati, la strategia di addizione non era entrata, per la Società Europea di Cardiologia questa è una delle strategie, ma si potrebbe continuare ad andare avanti rispetto a terapie elettriche e ad altro. Credo che realisticamente non dovremo rinunciare al fatto che nell'impostazione della terapia dello scompenso cardiaco non dobbiamo solo controllare il rimodellamento ventricolare, correggere il rischio di una morte improvvisa da substrato strutturato, ma è fondamentale la prevenzione dei nuovi eventi che si realizza attraverso gli ACE-inibitori e attraverso i betabloccanti, che riducono per esempio le recidive infartuali, ma si realizza anche attraverso le antiaggregazioni appropriate, attraverso la correzione del profilo di rischio cardiovascolare globale. Quindi, nel percorso di cura dei pazienti con scompenso cronico, che pure abbiano superato il loro vecchio infarto e che non abbiano angina, se c'è sotto una cardiopatia coronarica, non potremo rinunciare a questo tipo di approccio, anche se per alcune componenti si attendono ancora le evidenze (CIBIS III). Abbiamo certamente incrementato l'impiego dei sartanici, ma non lo abbiamo fatto in termini di strategie di addizione, che non è ancora incorporata nelle scelte cliniche, né della comunità internazionale, infatti l'indicazione per l'American Heart Association è solo di classe 2b [3], ugual- mente la Società europea di Cardiologia [2], che pure dà uno spazio importante, distingue le raccomandazioni di classe 1 sulle ospedalizzazioni da quelli sulla mortalità, di classe $2^{\circ}$.

Questo è probabilmente un mutamento strategico del quale dovremo ricordarci insieme a tutti gli enormi passi in avanti che abbiamo fatto sul betablocco come strategia dei pazienti con scompenso cardiaco, e i percorsi, in termini di quali strategie terapeutiche, per quali pazienti, nel campo del betablocco, nel mondo reale, continueranno a porre il problema di: quale farmaco? Su questo punto, la discrezione del singolo clinico continuerà ad essere ampia rispetto alle molecole proposte dalle linee guida, nebivololo, metoprololo succinato, che non è in commercio in Italia, carvedilolo, bisoprololo. Quale betabloccante? È un problema che esiste nella vita di tutti i giorni e probabilmente una conferenza nazionale può anche trasferire il parere di alcuni professionisti su questa problematica. Quando iniziare il betablocco? Come ha accennato il Prof. Metra, si tende sempre di più ad anticipare l'inizio della terapia con betablocco perché questa consente di capitalizzare in numero di pazienti trattati e in dose assunta nel follow-up. Quanto farmaco dare ai pazienti? È vero che più si bradicardizza i pazienti più si raggiunge l'endpoint di mortalità, però, anche in coloro in cui si bradicardizzava poco con una dose più bassa, o nei quali addirittura la frequenza cardiaca non si modificava ma incrementava, comunque c'era un impatto sulla sopravvivenza. Quindi, in questo contesto, nella popolazione anziana, anche basse dosi potrebbero sortire l'effetto in termini di endpoint hard. Si comincia prima il betablocco o gli ace-inibitori? Il CIBIS sembra suggerire che si possa cominciare prima con il betablocco. A mio avviso, nel mondo reale, noi continueremo a stabilizzare i nostri pazienti ottimizzando più rapidamente le dosi di ACE e di diuretico e aggiungeremo il betablocco che, in verità, in alcuni pazienti abbastanza selezionati, potrebbe essere cominciato come farmaco fin dall'inizio, ma parliamo in genere di disfunzioni ventricolari pauci-sintomatiche postinfartuali o di pazienti spiccatamente tachicardici senza avere sintomi da congestione etc.

L'esercizio fisico per la Società Europea di Cardiologia ha uno spazio importante, con raccomandazione 1 e livello di evidenza $b$, ricordando gli effetti in termini di capacità di esercizio e sicurezza, non ce l'ha in termini di mortalità perché non abbiamo studi sufficienti e la stessa posizione tende a riprodursi per l'American College e American Heart [3], che hanno un livello di evidenza $\mathrm{b}$ ma, una raccomandazione di classe 1 nei pazienti che abbiano depressione della funzione sistolica e abbiano avuto, o continuino ad avere, sintomi. Non ho personalmente letto i risultati di questo trial ma mi risulta che c'è uno studio randomizzato in corso. L'area della terapia non-farmacologica pone un problema serio di appropriatezza nella selezione dei pazienti e di razionale uso delle risorse; su questo aspetto credo che i professionisti dovranno essere molto sensibili. Questo tipo di conferenze nazionali devono avere il coraggio di sollevare il problema di quanti sono i teorici pazienti candidati, e quanti sono i pazienti per i quali è realmente sostenibile una terapia di questo ti- 
po. Questo evoca però la necessità di analizzare complessità, specificità di malati, profili di comorbidità, analizzare quanto investire sul maggior rischio di morte improvvisa, e quale è l'attesa di vita stimata dei nostri pazienti per produrre una scelta che sia la più razionale. Cambiamo argomento: è sufficiente che un paziente abbia vasi graftabili o miocardio genericamente vitale, perché nel modello della cardiopatia coronarica scompensata egli debba essere rivascolarizzato chirurgicamente? La risposta è che nel mondo reale non esiste solo la "graftabilità", esistono i volumi, l'ipertensione polmonare, il ruolo del ventricolo destro, e il profilo di comorbidità dei pazienti. Nei percorsi di cura di alcuni dei nostri pazienti selezionati, questo tipo di terapia non farmacologica esiste, ma richiede capacità di selezione ed analisi delle complessità (si pensi alla cosiddetta destination therapy con dispositivi di assistenza ventricolare).

Infine esiste anche l'ambito della fase terminale dello scompenso cardiaco e delle terapie palliative, così come c'è l'ambito della gestione del lutto, dell'economia del morire di una malattia che è destinata a coinvolgere sempre più frequentemente persone anziane in età avanzata. Quindi l'ultima delle quattro aree, è l'area dell'organizzazione, l'unica che potrà garantire di capitalizzare nel lungo termine efficacia di trattamenti per i nostri pazienti. Questo credo che sia eticamente dovuto, perché, così come non è accettabile, come Galli ricordava, non avere un approccio globale al rischio cardiovascolare in un paziente che sia stato rivascolarizzato (aspetto che potrebbe essere la maggior determinante nel lungo termine, superata la fase acuta), allo stesso modo non ha senso dispensare una terapia sapientemente e at- tentamente costruita, se non c'è chi si fa carico di questi pazienti e ne sa seguire il destino, aggiustare le dosi, aggiornare la terapia monitorare stili di vita ed aderenza alle terapia. Ci aiuterà la proteonomica e la farmacogenomica a selezionare meglio i nostri pazienti? Non abbiamo ancora sufficienti elementi per incorporare raccomandazioni in un documento di consenso nazionale. L'assunzione responsabile di scelte cliniche nella vita di tutti i giorni può essere difficile quando bisogna accreditare un peso agli obiettivi quantità vs qualità di vita. Facciamo un lavoro difficile, nel quale si può sbagliare, ma, come diceva Popper: "Evitare errori è un ideale meschino: se non osiamo affrontare problemi che siano così difficili da risolvere da rendere questo errore quasi inevitabile; non ci sarà allora sviluppo della conoscenza. In effetti, è dalle nostre teorie più ardite, incluse quelle che sono erronee, che noi impariamo di più. Nessuno può evitare di fare errori; la cosa grande è imparare da essi”.

\section{Bibliografia essenziale}

1. Komajda M., Lapuerta P, Hermans N, et al. Adherence to guidelines is a predictor of outcome in chronic heart failure: the Mahler survey. Eur Heart J 2005; 26; 16531659.

2. The Task Force for the Diagnosis and treatment of Chronic Heart Failure of the European Society of Cardiology; Guidelines for the Diagnosis and treatment of chronic heart failure: executive summary (update 2005). Eur Heart J 2005; 26; 1115-1140.

3. Hunt SA, Abraham WT, Chin MH, et al. ACC/AHA 2005 Guidelines Update for the Diagnosis and Management of Chronic Heart failure in the Adult. $J$ Am Coll Cardiol 2005; 46; 1116-1143. 